\title{
BIOLOGÍA REPRODUCTIVA DE Rhodophiala bifida (Amaryllidaceae): ASPECTOS DE APLICACIÓN EN EL MEJORAMIENTO GENÉTICO
}

\author{
Facciuto, G. ${ }^{1}$; Coviella, A. ${ }^{1} \&$ Bugallo, ${ }^{1}$.
}

\begin{abstract}
RESUMEN
Rhodophiala bifida es una especie bulbosa nativa de Argentina, Brasil, Paraguay y Uruguay. El tamaño y color de sus flores la hacen muy atractiva para el cultivo ornamental. El desarrollo de protocolos de domesticación y mejoramiento genético son esenciales para poder proponerla como un nuevo cultivo, considerando que la industria florícola global requiere novedades. Como punto de partida, se hicieron estudios de compatibilidad y eficiencia reproductiva a través de la realización de cruzamientos controlados considerando fenotipos de flores de distintos colores.

Tanto la autopolinización como la polinización cruzada produjeron frutos por lo que se comprobó un sistema reproductivo mixto en R. bifida. La eficiencia reproductiva fue significativamente mayor en la polinización cruzada que en la autopolinización siendo 49.6 y 19 respectivamente, para las flores rojas, genotipo más representativo de la población. No se observó fructificación en flores emasculadas sin polinizar por lo que se descartó la apomixis autónoma. Los cruzamientos entre fenotipos produjeron frutos excepto para el caso de cruzamientos entre fenotipos de flores rosas por fucsias.
\end{abstract}

Palabras clave: autocompatibilidad, eficiencia reproductiva, plantas nativas de Argentina, ornamentales, mejoramiento genético.

1.- Instituto de Floricultura. INTA Castelar. Los Reseros y Las Cabañas s/n. Castelar, Buenos Aires, Argentina. Email: facciuto.gabriela@inta.gob.ar

Manuscrito recibido el 6 de abril de 2020 y aceptado para su publicación el 22 de junio de 2020.

Facciuto, G.; Coviella, A.; Bugallo, V. Biología reproductiva de Rhodophiala bifida (Amaryllidaceae): aspectos de aplicación en el mejoramiento genético. FAVE - Ciencias Agrarias 20 (1): 147-157.

CC BY-NC-SA 4.0 


\begin{abstract}
Reproductive biology of Rhodophiala bifida (Amaryllidaceae): application aspects in breeding.

Rhodophiala bifida is a bulbous species native to Argentina, Brazil, Paraguay and Uruguay. The size and color of its flowers make it very attractive for ornamental cultivation. The development of domestication and breeding protocols are essential to propose it as a new crop, considering that the global floricultural industry requires novelties. As a starting point, compatibility and reproductive efficiency studies were carried out through controlled crosses considering phenotypes of flowers of different color. Both self-pollination and cross-pollination produced fruits, so a mixed reproductive system was verified in R. bifida. Reproductive efficiency was significantly higher in cross-pollination than in self-pollination, being 49.6 and 19 respectively, for red flowers, the most representative genotype of the population. No fruiting was observed in emasculated flowers without pollination, so autonomous apomixis was ruled out. The crossings among phenotypes produced fruits except for the case of crossings between pink by fuchsias flowered phenotypes.
\end{abstract}

Key words: selfcompatibility, reproductive efficiency, plants native to Argentina, ornamentals, breeding.

\section{INTRODUCCION}

Rhodophiala bifida (Herb.) Traub - azucenita colorada- es una especie bulbosa perteneciente a la familia Amaryllidaceae, nativa de Argentina, Brasil, Paraguay y Uruguay (Arroyo-Leuenberger, 1996). Esta familia se destaca por sus numerosos géneros cultivados como plantas ornamentales tales como Amaryllis, Crinum, Galanthus, Leucojum, Lycoris y Narcissus (Simpson, 2006).

En Argentina, $R$. bifida se distribuye en las provincias de Buenos Aires, Entre Ríos, Corrientes y Misiones en pastizales de suelos fértiles y bien drenados (Hurrell et al., 2009). Es una especie perenne de floración estival tardía que se concentra luego de las lluvias. Las inflorescencias están formadas por 2 a 7 flores zigomorfas, infundibuliformes, declinadas y pediceladas. Sus flores presentan 6 tépalos de color rojo carmín, bermellón, rojo vinoso, rosado y rara vez blanco (Hurrell et al., 2009).
El tamaño y color de sus flores la hacen muy atractiva para el cultivo ornamental. Además, se propone especialmente para xerojardinería, ya que tiene bajo requerimiento hídrico y nutricional que la hace apta para jardines con un uso racional del agua, aspecto muy valorado en tiempos en que se busca mitigar los efectos del cambio climático (Bisgrove y Hadley, 2002). El desarrollo de protocolos de domesticación, propagación y mejoramiento genético son esenciales para poder proponerla como un nuevo cultivo, considerando que la industria florícola global requiere novedades.

En Amaryllidaceae se encuentran especies con distintos grados de compatibilidad (Broyles y Wyatt, 1991; Fernandez et al., 2013; Saavedra et al., 1996; Sage et al., 1999) e incompatibilidad (Streher et al., 2018). En algunos casos, se evidenció autoincompatibilidad de acción tardía (Kiepiel y Johnson, 2014; Vaughton et al., 2010). A pesar de ello, la mayoría de los

Revista FAVE - Ciencias Agrarias 20 (1) 2021 
sistemas reproductivos de las plantas de la familia siguen siendo desconocidos, especialmente en las especies del hemisferio sur, donde se encuentran la mayor diversidad (Kiepiel y Johnson, 2014).

La comparación de los resultados en autopolinización y polinización cruzada es uno de los tópicos centrales en los estudios de biología reproductiva y resulta esencial su abordaje antes de comenzar tareas de mejoramiento genético. Asimismo, el estudio del crecimiento del tubo polínico en las polinizaciones controladas puede aportar información acerca de las posibles barreras que dificulten la hibridación.

El objetivo de este trabajo fue estudiar la compatibilidad y eficiencia reproductiva en $R$. bifida a través de la realización de cruzamientos controlados, tanto autopolinizaciones como polinizaciones cruzadas, tomando en cuenta fenotipos de flores de distintos colores como inicio de un plan mejoramiento genético para la obtención de variedades ornamentales.

\section{MATERIALES Y MÉTODOS}

\section{Material vegetal}

Se utilizaron para los experimentos 400 plantas de una población de $R$. bifida naturalizada en el Jardín Botánico Arturo Ragonese, Instituto de Recursos Biológicos INTA, Partido de Hurlingham, Buenos Aires, Argentina ( $34^{\circ} 40 \mathrm{~S}, 5^{\circ} 39 \mathrm{O}, 22 \mathrm{msnm}$ ) donde se hallaron fenotipos de tres colores de flores: rojas, fucsias y rosas con predominancia de color rojo (Fig. 1). No se hicieron cuidados culturales de la población excepto el corte de pastizales en verano.

Los suelos del predio son arguidoles típicos (Gomez, 1993) y según la serie histó- rica del observatorio Agrometeorológico de INTA Castelar, Instituto de Clima y Agua, la temperatura máxima y mínima media anual en la zona es de $30^{\circ} \mathrm{C}$ y $17^{\circ} \mathrm{C}$, respectivamente. La precipitación anual aproximada es de $1000 \mathrm{~mm}$. La humedad relativa anual varía entre 64 y $79 \%$.

\section{Cruzamientos}

Los cruzamientos se realizaron en febrero y marzo de 2014 y 2018 entre las 10 y 14 hs. Para ello, se emascularon flores en estado de botón cerrado de un tamaño de 3 a $5 \mathrm{~cm}$ y se procedió a la polinización con granos de polen de la misma planta o de plantas distintas según el caso. Para la polinización cruzada se utilizaron individuos distantes en la población. Luego de la polinización, las flores se cubrieron con sobres de papel para evitar la contaminación con polen extraño. También, se consideraron flores emasculadas y sin polinizar con el fin de observar una posible partenogénesis.

En un primer experimento (2014) se realizaron cruzamientos sólo con plantas de flores rojas, color de mayor representatividad en la población. Los tratamientos fueron autopolinización, polinización cruzada y flores emasculadas sin polinizar.

En el segundo experimento (2018), se profundizó en las diferencias fenotípicas y se consideraron plantas con flores las rojas, fucsias y rosas. A los tratamientos antes mencionados se sumó la fructificación libre, es decir, se consideraron flores marcadas sin ningún tratamiento para identificar la fructificación natural.

Se evaluó en ambos casos la cantidad de frutos producidos en los cruzamientos y se calculó el porcentaje de fructificación (Fructificación $\%=$ frutos producidos/flores polinizadas x 100). Se evaluó la cantidad de se- 


\section{G. Facciuto et al.}

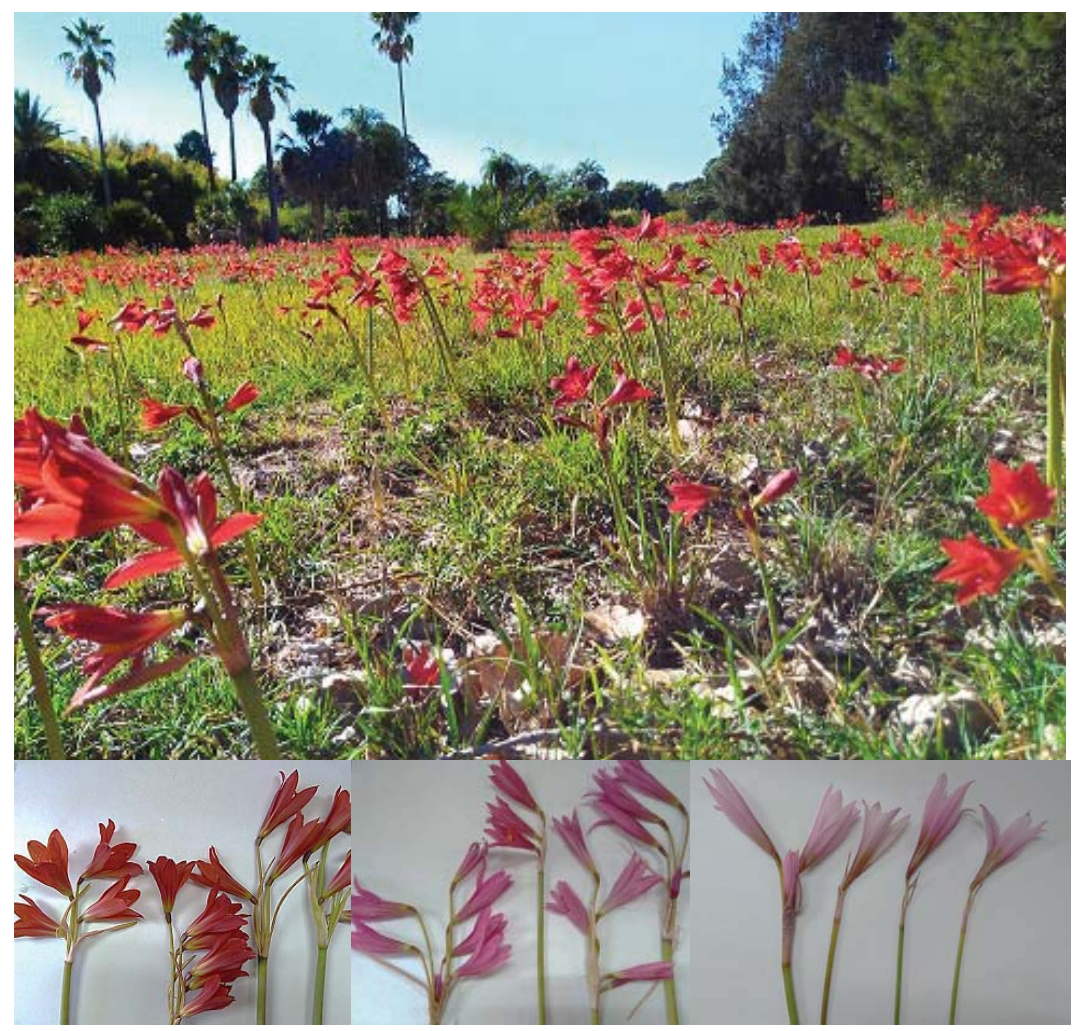

Figura 1: Población de Rhodophiala bifida del Jardín Botánico Arturo Ragonese, Instituto de Recursos Biológicos INTA (arriba). Fenotipos de flores rojas, fucsias, rosas (de izquierda a derecha, abajo). Figure 1: Population of Rhodophiala bifida of the Botanical Garden Arturo Ragonese, Instituto de Recursos Biológicos INTA (above). Phenotypes of red, fuchsia, pink flowers (from left to right, below).

millas obtenidas por fruto y su germinación, para lo cual las semillas se colocaron en cajas plásticas sobre papel de filtro (3) y se mantuvieron en cámara a $25 \pm 2^{\circ} \mathrm{C}$ y con un fotoperíodo de 16 horas luz/ 8 horas oscuridad. A los 14 días de la siembra, se procedió al conteo de las plántulas normales obtenidas.

La eficiencia reproductiva se calculó según la fórmula de Dafni (1992): ( $\mathrm{N}^{\circ}$ frutos/ $\mathrm{N}^{\circ}$ flores polinizadas) $\mathrm{x}\left(\mathrm{N}^{\mathrm{o}}\right.$ promedio de semillas por fruto/ $\mathrm{N}^{\mathrm{o}}$ promedio óvulos/ ovario) y se le agregó el factor $\mathrm{N}^{\mathrm{o}}$ plántulas normales/ $\mathrm{N}^{\circ}$ semillas sembradas. El número promedio de óvulos por ovario se calculó contando todos los óvulos de 15 ovarios sobre lupa.
El índice de autocompatibilidad (IAI) se obtuvo como el cociente entre el porcentaje de frutos producidos por autopolinización y el porcentaje obtenido por polinización cruzada según Ruiz y Arroyo (1978).

Los datos se sometieron a análisis estadísticos a través de tests de diferencia de proporciones y ANOVA $(\mathrm{p} \leq 0.05)$ con el programa INFOSTAT 2009 (Di Rienzo et al., 2011).

\section{Crecimiento del tubo polínico}

Para evaluar la velocidad y reacción de los tubos polínicos en los cruzamientos, se recolectaron pistilos al primero, segundo y tercer día posterior a las polinizaciones Revista FAVE - Ciencias Agrarias 20 (1) 2021 
(DPP), tanto en la autopolinización como en polinización cruzada. Se fijaron en FAA (formol; ácido acético; etanol/1: 0,5:8) por, al menos, 24 horas. Luego, se enjuagaron con agua destilada y se trataron con $\mathrm{NaOH}$ $8 \mathrm{~N}$ a $60^{\circ} \mathrm{C}$ por dos horas según el método de Martin (1959). Los pistilos se enjuagaron nuevamente, se tiñeron con azul de anilina al $1 \%$ en agua durante una hora y se montaron sobre un portaobjetos con una gota de la mencionada solución, se cubrieron con un cubreobjetos y se presionó levemente. Se observaron con microscopio de epi-fluorescencia OLYMPUS BX 50 (Japón) con filtros de excitación y emisión de 330-385 $\mathrm{nm}$ y $420 \mathrm{~nm}$, respectivamente. Se evaluó el crecimiento del tubo polínico en los pistilos considerando la distancia recorrida y a los 3 DPP se contabilizó la cantidad de tubos polínicos que llegaron al ovario. Se evaluaron 10 pistilos por tratamiento.

\section{RESULTADOS}

Los cruzamientos derivados de la autopolinización y la polinización cruzada produjeron frutos en $R$. bifida. En la Tabla 1 se presentan los datos de fructificación, producción de semillas por fruto y plántulas obtenidas en la auto-polinización y en la polinización cruzada en los cruzamientos realizados utilizando flores rojas, genotipo predominante en la población estudiada (experimento 1). La capacidad de producir frutos fue significativamente mayor en la polinización cruzada respecto a la autopolinización siendo de $79.6 \%$ y $52.6 \%$, respectivamente. Dicha tendencia también se reflejó en los valores de eficiencia reproductiva siendo 19 y 49.6 para la autopolinización y polinización cruzada, respectivamente. En ningún caso las flores emasculadas y no polinizadas produjeron frutos.

Tabla 1: Fructificación (\%), semillas por fruto (prom.) y eficiencia reproductiva en la autopolinización y polinización cruzada de Rhodophiala bifida realizado con fenotipos de flores rojas. Table 1: Fructification (\%), seeds per fruit (average) and reproductive efficiency in selfpollination and cross-pollination of Rhodophiala bifida with phenotypes of red flowers.

\begin{tabular}{|l|c|c|c|}
\hline & Autopolinización & $\begin{array}{c}\text { Polinización } \\
\text { cruzada }\end{array}$ & $\begin{array}{c}\text { Flores emasculadas } \\
\text { sin polinizar }\end{array}$ \\
\hline Flores polinizadas No & 38 & 49 & 13 \\
\hline Frutos obtenidos N & 20 & 39 & 0 \\
\hline Fructificación \% & $52.6 \mathrm{a}$ & $79.6 \mathrm{~b}$ & 0 \\
\hline IAl 0.66 & $26 \pm 14.3$ & $41.4 \pm 25.4$ & - \\
\hline Semillas por fruto prom. & $208 / 116$ & $663 / 481$ & \\
\hline $\begin{array}{l}\text { Semillas } \\
\text { sembradas/plántulas } \\
\text { normales No }\end{array}$ & $19 \mathrm{~b}$ & $49.6 \mathrm{a}$ & - \\
\hline Eficiencia reproductiva & & & \\
\hline
\end{tabular}

Letras diferentes indican diferencias significativas en test de diferencia de proporciones para la cantidad de frutos obtenidos en las polinizaciones y eficiencia reproductiva. 
G. Facciuto et al.

Tabla 2: Fructificación (\%), semillas por fruto (prom.), indice de autoincompatibilidad y eficiencia reproductiva en la auto-polinización y polinización cruzada de Rhodophiala bifida en fenotipos de flores rojas, fucsias y rosas.

Table 2: Fructification (\%), seeds per fruit (average), selfincompatibility index and reproductive efficiency in self-pollination and cross-pollination of Rhodophiala bifida in phenotypes with red, fuchsia and pink flowers.

\begin{tabular}{|c|c|c|c|c|}
\hline & Autopolinización & $\begin{array}{c}\text { Polinización } \\
\text { cruzada }\end{array}$ & $\begin{array}{l}\text { Emasculación sin } \\
\text { polinización }\end{array}$ & $\begin{array}{c}\text { Fructificación } \\
\text { libre }\end{array}$ \\
\hline \multicolumn{5}{|l|}{ Flores Rojas } \\
\hline Flores polinizadas $\mathrm{N}^{\circ}$ & 46 & 41 & 10 & 37 \\
\hline Frutos obtenidos $\mathrm{N}^{\circ}$ & $29 a$ & $29 \mathrm{a}$ & $0 \mathrm{~b}$ & $29 \mathrm{a}$ \\
\hline $\begin{array}{l}\text { Fructificación \% } \\
\text { IAl } 0.89\end{array}$ & $63.04 \mathrm{a}$ & $70.73 \mathrm{a}$ & 0 & 78.37 \\
\hline Semillas por fruto & $29.97 \mathrm{a}$ & $48.96 \mathrm{~b}$ & 0 & $47.17 \mathrm{~b}$ \\
\hline Eficiencia reproductiva & $25.84 \mathrm{~b}$ & $47.35 \mathrm{a}$ & 0 & $50.53 a$ \\
\hline $\begin{array}{l}\text { Plántulas obtenidas/ } \\
\text { Semillas sembradas }\end{array}$ & $646 / 820$ & $692 / 893$ & 0 & $610 / 707$ \\
\hline E.R. modificada & 20.35 & 36.69 & 0 & 43.59 \\
\hline \multicolumn{5}{|l|}{ Flores Fucsias } \\
\hline Flores Polinizadas $\mathrm{N}^{\circ}$ & 27 & 32 & 15 & 45 \\
\hline Frutos obtenidos $\mathrm{N}^{\mathrm{O}}$ & $1 \mathrm{c}$ & $13 \mathrm{~b}$ & $0 \mathrm{c}$ & $32 \mathrm{a}$ \\
\hline $\begin{array}{l}\text { Fructificación \% } \\
\text { IAI } 0.09\end{array}$ & 3.7 & 40.62 & 0 & 71.11 \\
\hline Semillas por fruto $\mathrm{N}^{\circ}$ & $7 \mathrm{a}$ & $18 \mathrm{~b}$ & 0 & 35.41 \\
\hline Eficiencia reproductiva & $0.34 \mathrm{c}$ & $9.71 \mathrm{~b}$ & 0 & $33.44 \mathrm{a}$ \\
\hline $\begin{array}{l}\text { Plántulas obtenidas/ } \\
\text { Semillas sembradas № }\end{array}$ & $7 / 7$ & $91 / 185$ & 0 & $627 / 1130$ \\
\hline E.R. modificada & 0.34 & 4.78 & 0 & 18.55 \\
\hline \multicolumn{5}{|l|}{ Flores Rosas } \\
\hline Flores polinizadas $\mathrm{N}^{\circ}$ & 7 & 13 & 11 & 40 \\
\hline Frutos obtenidos $\mathrm{N}^{\circ}$ & $1 \mathrm{~b}$ & $6 \mathrm{~b}$ & $0 \mathrm{c}$ & $34 \mathrm{a}$ \\
\hline Fructificación \% & 14.28 & 46.15 & 0 & 85.00 \\
\hline \multicolumn{5}{|l|}{ IAI 0.30} \\
\hline Semillas por fruto $\mathrm{N}^{\circ}$ & $14 \mathrm{a}$ & $36.8 \mathrm{~b}$ & 0 & 33.95 \\
\hline Eficiencia reproductiva & $2.11 \mathrm{c}$ & $17.93 \mathrm{~b}$ & 0 & $30.47 \mathrm{a}$ \\
\hline $\begin{array}{l}\text { Plántulas } \\
\text { obtenidas/semillas } \\
\text { sembradas } \mathrm{N}^{0}\end{array}$ & $12 / 14$ & $54 / 105$ & 0 & $437 / 645$ \\
\hline E. R. modificada & 1.81 & 9.22 & 0 & 20.64 \\
\hline
\end{tabular}

Letras diferentes indican diferencias significativas en test de diferencia de proporciones para la cantidad de frutos obtenidos en las polinizaciones y eficiencia reproductiva según el color de flor de la planta madre. En semillas por fruto letras diferentes indican diferencias significativas ANOVA $p \leq 0.05$.

Valores de óvulos/ovario para cálculo de eficiencia reproductiva: flores rojas $73.33 \pm 16.92$, flores fucsias

$75.23 \pm 7.8$, flores rosas $95 \pm 12.19$. 
En el segundo experimento se produjeron frutos tanto en la autopolinización como en la polinización cruzada considerando los tres fenotipos florales (Tabla 2). El porcentaje de fructificación fue mayor en la polinización entre los fenotipos de flores fucsias y entre los de flores rosas; las plantas de flores rojas no presentaron diferencias significativas. A pesar de esta excepción, los valores de semillas por fruto fueron significativamente mayores en la polinización cruzada en los tres fenotipos (Tabla 2). Los datos de IAI para los fenotipos de flores rojas, fucsias y rosas, fueron $0.89,0.09$ y 0.30 respectivamente (Tabla 2).

No hubo diferencias significativas en la fructificación natural entre los tres fenotipos para color de flor; el porcentaje de formación de frutos en flores marcadas sin polini- zar manualmente arrojó valores de 78,37\%, $85 \%$ y $71,11 \%$ para fenotipos de flores rojas, rosas y fucsias, respectivamente (Tabla 2).

El estudio del crecimiento de los tubos polínicos en los pistilos autopolinizados y sometidos a polinización cruzada para el caso de flores rojas demostró que, a los 3 DPP, éstos llegan a los ovarios en ambos casos. En la Fig. 2 se graficaron la cantidad de muestras observadas (\%) y la cantidad de tubos polínicos observados. Debido al elevado número de tubos polínicos y a su superposición resultó metodológicamente imposible realizar un recuento de los mismos cuando superaban los 100 tubos. A pesar de ello, se observó una diferencia muy notoria ya que se contabilizaron hasta 20 y más de 100 tubos polínicos en el ovario de pistilos autopolinizados y sometidos a polinización

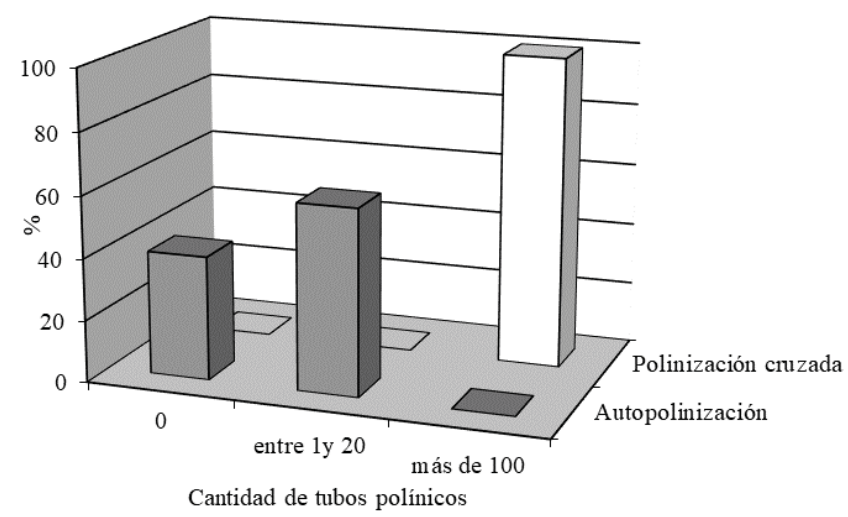

Figura 2: Tubos polínicos (\%) observados en el ovario en pistilos autopolinizados y sometidos a polinización cruzada de Rhodophiala bifida a los 3 dias post polinización considerando la cantidad de los mismos (flores rojas).

Figure 2: Pollen tubes (No.) observed in the ovary in self-pollinated pistils and cross-pollination of Rhodophiala bifida 3 days after pollination considering the amount of them (red flowers). 


\section{G. Facciuto et al.}

cruzada, respectivamente. En la Fig. 3 se presentan fotografías de ambas situaciones observando estas conspicuas diferencias.

Los cruzamientos entre fenotipos de distintos colores produjeron frutos en todos los casos excepto para el caso de los cruzamientos entre genotipos de flores rosas $\times$ fucsias (Tabla 3). La eficiencia reproductiva más alta (21.66) se presentó en fenotipos de flores rojas polinizados por fenotipos de flores rosas.
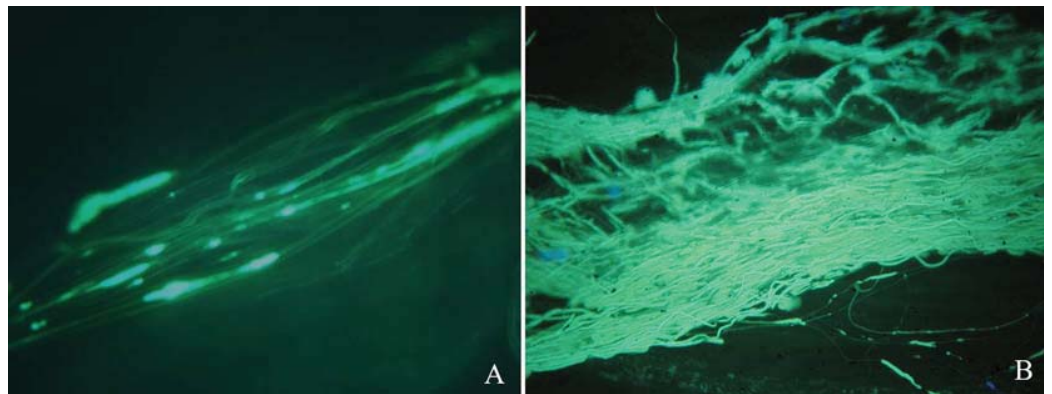

Figura 3: Crecimiento de tubos polínicos en el estilo en la autopolinización (izquierda) y polinización cruzada de Rhodophiala bifida (flores rojas) a los 3 días post polinización.

Figure 3: Growth of pollen tubes in the style in self-pollination (left) and crosspollination of Rhodophiala bifida (red flowers) at 3 days post pollination.

Tabla 3: Cruzamientos entre los fenotipos de flores rojas, fucsias y rosas de Rhodophiala bifida. Table 3: Crossings among phenotypes with red, fuchsia and pink flowers of Rhodophiala bifida.

\begin{tabular}{|c|c|c|c|c|c|c|c|}
\hline Fenotipos & 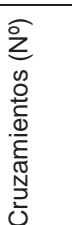 & 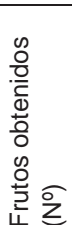 & 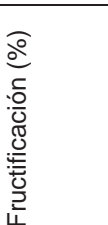 & 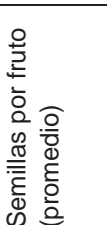 & 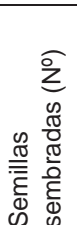 & 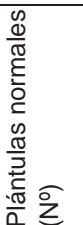 & 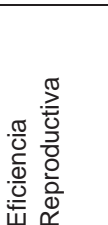 \\
\hline Roja $\times$ Fucsia & 8 & 1 & $12.50 \mathrm{a}$ & 5.00 & 5 & 4 & $0.68 \mathrm{~b}$ \\
\hline Roja $\times$ Rosa & 25 & 13 & $52.00 a$ & 36.46 & 474 & 395 & $21.66 \mathrm{a}$ \\
\hline Fucsia × Roja & 11 & 2 & $18.18 a$ & 12.00 & 24 & 21 & $2.52 \mathrm{~b}$ \\
\hline Fucsia × Rosa & 22 & 1 & $4.54 \mathrm{~b}$ & 38.00 & 38 & 32 & $1.92 \mathrm{~b}$ \\
\hline Rosa $\times$ Fucsia & 7 & 0 & ob & 0 & 0 & 0 & 0 \\
\hline Rosa $\times$ Roja & 15 & 7 & $46.66 \mathrm{a}$ & 22.17 & 133 & 87 & $7.11 \mathrm{~b}$ \\
\hline
\end{tabular}

Letras diferentes indican diferencias significativas en test de diferencia de proporciones para la cantidad de frutos obtenidos y eficiencia reproductiva

Óvulos/ovario para cálculo de eficiencia reproductiva: flores rojas $73.33 \pm 16.92$, flores fucsias $75.23 \pm 7.8$, flores rosas $95 \pm 12.19$. 


\section{DISCUSIÓN}

A partir de los resultados de los cruzamientos dirigidos, germinación de las semillas y los estudios del crecimiento del tubo polínico se comprobó un sistema reproductivo mixto para $R$. bifida. A pesar de ello, la eficiencia reproductiva y la producción de semillas fueron menores en la autopolinización, tal como Ladd y Arroyo (2009) reportaron para $R$. rhodolirion. La autopolinización generó una menor producción de semillas que la polinización cruzada, esta característica también se ha presentado en otras especies de Amaryllidaceae tales como Narcissus triandrus (Sage et al., 1999) y Hippeastrum advenum (Saavedra et al., 1996) y Habranthus tubispathus (Fernandez et al., 2013). Para el caso de Zephyranthes atamasco, Broyles y Wyatt (1991) hallaron la misma cantidad de semillas en frutos provenientes de autopolinización que en la polinización cruzada. La longevidad de cada flor de $R$. bifida fue de aproximadamente 3 días (observación de las autoras), período que, por ser tan corto, requeriría de una polinización y fecundación eficientes como lo sugiere este caso al producir frutos y semillas viables, tanto en la autopolinización, como en la polinización cruzada.

Según Echeverría y Alonso (2010), las semillas de $R$. bifida presentarían nula a escasa dormición innata ya que germinan casi en su totalidad luego de dos meses de la cosecha. A partir de los resultados obtenidos por estos autores, se infiere que la dormición no estaría interfiriendo en la cantidad de plántulas normales obtenidas a partir de las semillas sembradas en los cruzamientos realizados en este trabajo.

Los estudios del crecimiento del tubo polínico en ambas situaciones (autopolinización y polinización cruzada) fueron consistentes con la cantidad de semillas obtenidas ya que, en la polinización cruzada la cantidad de tubos polínicos que arribaron al ovario fue significativamente mayor. Esta característica demostró que a pesar de que $R$. bifida produce semillas viables a partir, tanto de la autopolinización como de la polinización cruzada, resulta más eficiente cuando se producen por esta última. Se puede sugerir la presencia de una reacción de autoincompatibilidad débil, que se acentúa en el caso de los fenotipos de flores fucsias.

La autoincompatibilidad es un sistema de auto-reconocimiento genético que favorece el cruzamiento entre individuos diferentes en plantas con flores hermafroditas (Durand et al., 2020). Es una de muchas estrategias que se cree ha evolucionado en respuesta al efecto negativo de la depresión por endocría, causada por la expresión de alelos deletéreos en homocigotas (Charlesworth y Charlesworth, 1987). El mantenimiento de la autoincompatibilidad en una población, como podría haber sucedido en esta especie, es reflejo del balance entre las ventajas aportadas por la prevención de los efectos deletéreos de la endocría y sus desventajas, que radican en la limitación reproductiva que este mecanismo conlleva (Durand et al., 2020).

Los resultados de IAI obtenidos en este trabajo, 0.89 y 0.30 para plantas de flores rojas y rosas respectivamente la clasifican como autocompatible. Según Ruiz y Arroyo (1978) un IAI mayor o menor a 0.20 indica una especie autocompatible o autoincompatible, respectivamente.

Stevens (2001) afirma que, de acuerdo con la bibliografía tradicional, la presencia de especies con IAI intermedio es común. Radusky et al. (2010) analizaron el IAI de 1238 especies correspondientes a 144 familias y determinaron que la frecuencia de la 
distribución era fuertemente bimodal, aunque hallaron que una fracción sustancial tenía valores intermedios, como en el caso de la especie en estudio.

En el caso de las plantas con flores de color fucsia, el AIA se redujo dramáticamente, arrojando un resultado de 0.09, clasificándola como autoincompatible. Sin embargo, sería interesante estudiar un posible efecto pleiotrópico que asocie este fenotipo a una menor viabilidad reproductiva.

En todos los casos de polinización libre se obtuvieron frutos con semillas viables, esto indicaría que no hubo limitaciones en la llegada de polen o presencia de polinizadores.

Asimismo, el crecimiento del tubo polínico reflejó una inhibición precigótica parcial en pistilos autopolinizados que luego se manifestó en la menor cantidad de semillas producidas. Streher et al. (2018) afirmaron que la germinación de granos de polen en el estigma y su detención en el estilo, situación encontrada en este trabajo, representa un signo típico de autoincompatibilidad gametofítica.

En ningún caso se observó fructificación en flores emasculadas sin polinizar por lo que se descartó la posibilidad de apomixis autónoma en esta especie.

En este trabajo, la variabilidad encontrada en el color de flor sería originaria de la segregación alélica en la población, reflejando la heterocigosis de los individuos de una población alógama.

\section{CONCLUSIONES}

Se comprobó un sistema reproductivo mixto en $R$. bifida, es decir polinización cruzada y autopolinización. A pesar de ello, la eficiencia reproductiva fue mayor en la polinización cruzada por lo que la especie tiende a ser más alógama. Se comprobó una inhibición precigótica parcial en la autopolinización. Se obtuvieron frutos y semillas viables en cruzamientos entre distintos fenotipos. Asimismo, se pudo descartar la posibilidad de una apomixis autónoma.

El presente trabajo permitió recabar información básica sobre la biología reproductiva para poder iniciar un plan de mejoramiento genético en $R$. bifida.

\section{AGRADECIMIENTOS}

Las autoras agradecen al Jardín Arturo Ragonese del Instituto de Recursos Biológicos INTA por permitir el uso de la población de $R$. bifida y a la Técnica en Floricultura Natalia Sidañez por las tareas de acondicionamiento, recuento y germinación de semillas.

\section{REFERENCIAS}

Arroyo-Leuenberger S C. 1996. Amaryllidaceae. En Zuluoga y Morrone, Catálogo de las plantas vasculares de la Argentina. I. Missouri Botanical Garden. 90-100.

Bisgrove R, Hadley P. 2002. Gardening in the Global Greenhouse: The Impacts of Climate Change on Gardens in the UK. Informe técnico. Centre for Horticulture and Landscape, school of Plant Sciences, The University of Reading. 134 pp. 
Broyles S B, Wyatt R. 1991. The breeding system of Zephyranthes atamasco (Amaryllidaceae). Bull. of the Torrey Bot. Club 118(2): 137-140.

Charlesworth D, Charlesworth B. 1987. Inbreeding depression and its evolutionary consequences. Annual review of Ecology and Systematics 18: 237-268.

Dafni A. 1992. Pollination ecology, a practical approach. Oxford University Press, Oxford. $245 \mathrm{pp}$.

Di Rienzo JA, Casanoves F, Balzarini M G, Gonzalez L, Tablada M, Robledo CW. 2011. InfoStat, FCA, Universidad Nacional de Córdoba, Argentina. URL http://www. infostat. com. Ar

Durand E, Chantreau M, LeVeve A, Stetsenko R, Dubin M, Genete M, Llaurens V, Poux C, Roux C; Billiard S, Vekemans X, Castric V. 2020. Evolution of self-incompatibility in the Brassicaceae: Lessons from a textbook example of natural selection. Evolutionary applications 00: 1-19.

Echeverría M L, Alonso S I. 2010. Germinación y crecimiento inicial de Amarilidáceas nativas con potencial ornamental. Rev. Fac. Ciencias Agr. Univ. Nac. Cuyo 42 (1): 23-37.

Fernandez A C, Marinangeli P, Curvetto N, Facciuto G. 2013. Reproductive biology of Handroanthus tubispathus. Acta Hort. 1000: 183188.

Gómez L. 1993. Carta básica semidetallada de suelos. Complejo de Investigaciones Castelar, INTA, Provincia de Buenos Aires. INTA-CIRN, Instituto de Suelos, Buenos Aires, $114 \mathrm{p}$

Hurrell J A, Roitman G, Delucchi G. 2009. Amarillydaceae. En Flora Rioplatense. Sistemática, ecología y etnobotánica de las plantas vasculares rioplatenses. Parte 3 vol 4. Director J.A. Hurrell.

Kiepiel I A N, Johnson S D. 2014. Breeding systems in Clivia (Amaryllidaceae): late-acting self -incompatibility and its functional consequences. Bot. J. of the Linnean 175:155-168.
Ladd P G, Arroyo M T K. 2009. Comparisons of breeding systems between two sympatric species, Nastanthus spathulatus (Calyceraceae) and Rhodophiala rhodolirion (Amaryllidaceae), in the high Andes of central Chile. Plant Species Biol. 24: 2-10.

Martin F. 1958. Staining and observing pollen tubes in the styles by means of fluorescence. Stain Tech. 34:125-128.

Raduski A R, Haney E B, Igic B. 2011. The expression of self-incompatibility in angiosperms is bimodal. Evol. 66-4: 1275-1283.

Ruiz T Z, Arroyo M T K. 1978. Plant reproductive ecology of secondary deciduous forest in Venezuela. Biotropica 19: 221-230.

Saavedra F, Arroyo M T K, Hoffmann A J. 1996. The breeding system of Hyppeastrum advenum (Ker-Gawl.) Herb. (Amaryllidaceae), an endemic bulbous geophyte of the mediterranean-type climate region in central Chile. Botanische Jahrbücher fur Systematik, Pflanzengeschichte und Pflanzengeographie 118 (1):1-8.

Sage T L, Strumas F, Cole W W, Barrett S C H. 1999. Differential ovule development following self- and cross-pollination: the basis of self-sterility in Narcissus triandrus (Amaryllidaceae). A. J. of Bot. 86:855-870.

Simpson M G 2006. Plant Systematics. Amaryllidaceae. Elsevier Academic Press. Canada. pp. 169-171.

Stevens PF. 2001. Angiosperm phylogeny website. Version 14, accedido 28 Julio 2017. http:// www.mobot.org/MOBOT/research/APweb/.

Streher N S, Guerra E, Lüdtke R, Semir J, Dutilh J H A. 2018. Self-incompatibility in Habranthus gracilifolius (Amaryllidaceae): pre- and post-pollination barriers. Braz. J. of Bot. 41(2) 375-384.

Vaughton G, Ramsey M, Johnson S D. 2010. Pollination and late acting self-incompatibility in Cyrtanthus breviflorus (Amaryllidaceae): implications for seed production. Annals of Bot. 106 (4): 547-555. 